\title{
AOTrauma D-A-CH 3-Länder-Tagung
}

Ulrich Stöckle, Karl-Heinz Frosch

Vom 09. bis 11. Mai 2019 fand in Hamburg die AOTrauma Deutschland-AustriaSchweiz (D-A-CH) 3-Länder-Tagung mit fast 200 Mitgliedern und Gästen statt. Zum ersten Mal nahmen Vertreter der OP-Personal-Teams teil. Auf der Mitgliederversammlung der deutschen AOTrauma wurden die Jahresbilanz 2018 gezogen und diverse Ehrungen vorgenommen.

Das wissenschaftliche Programm der Tagung war geprägt von intensiven Falldiskussionen in den Bereichen periprothetische Frakturen, komplexe Verletzungen des Schultergürtels sowie Komplikations- und Infektmanagement. In der Sitzung „Neue Lehrformate“ wurden neue AO-Programme wie „Blended Learning“ (eLearning, Touch Surgery...) und das „Competency Training \& As- sessment Program“ vorgestellt sowie Kursformate mit realitätsnaher Frakturversorgung diskutiert. Sowohl der Past-Präsident, Klaus Renner, als auch der Präsident-elect der AOF, Florian Gebhard, informierten über Neuigkeiten aus der AO und Michael Raschke aus dem TK-System. Die Teilnehmer konnten im Laufe der Tagung unter Anleitung von Experten der AOT Education und Community Deve-

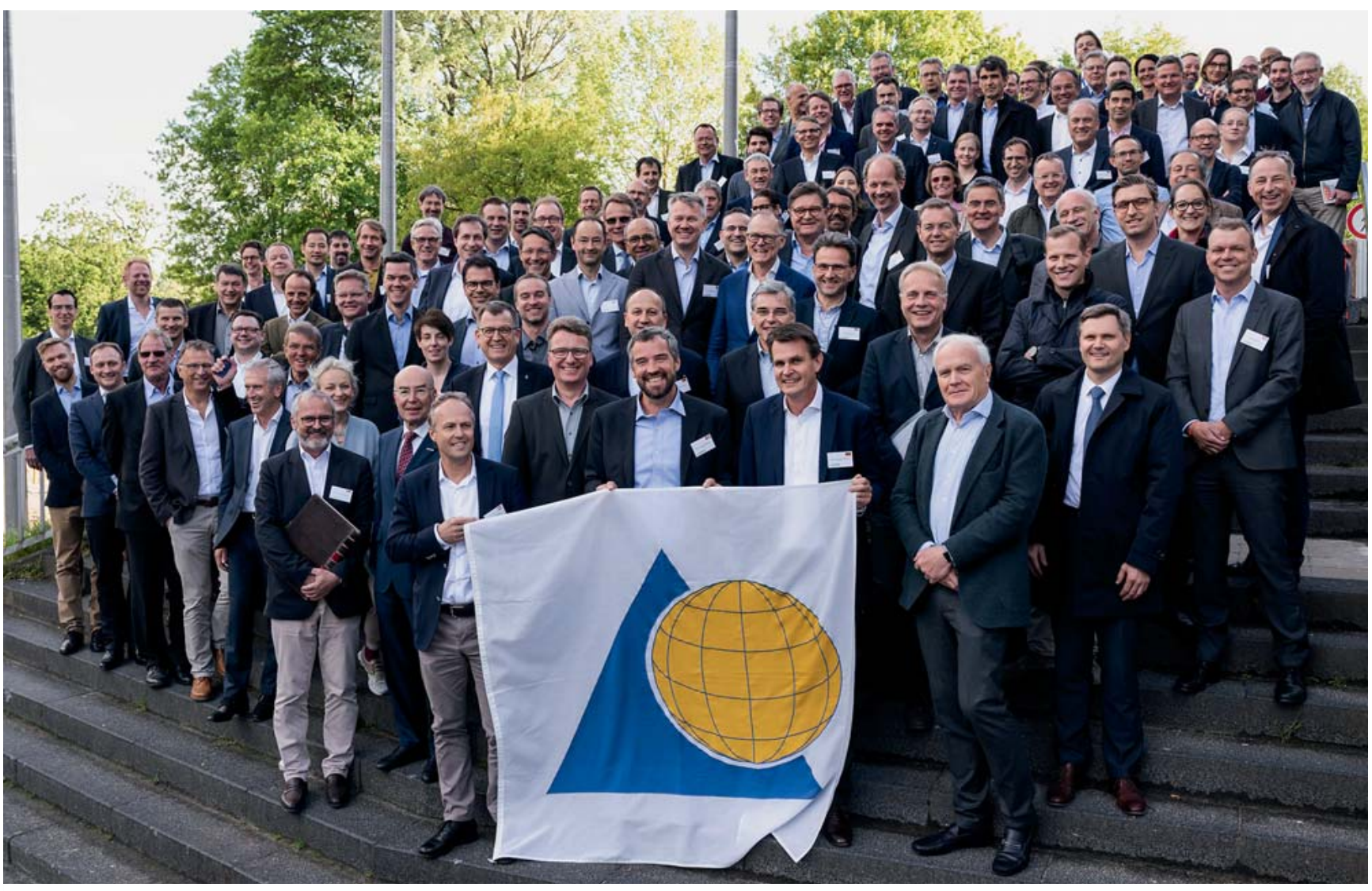

- Abb. 1 Teilnehmer der AOT-3-Länder-Tagung. Quelle: AOTrauma 


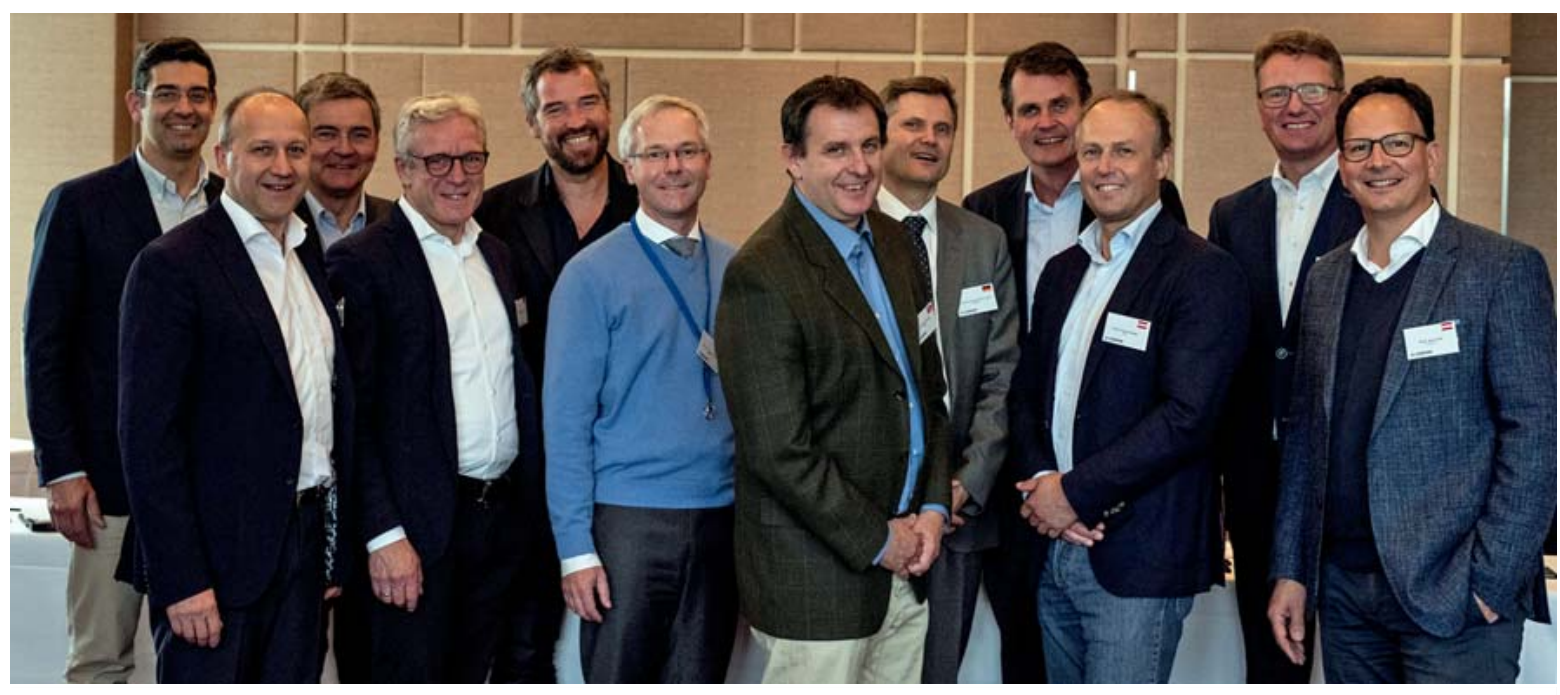

Abb. 2 Gemeinsame Sitzung der Präsidien von Deutschland, Österreich und Schweiz. Quelle: AOTrauma

lopment die verschiedenen Programme und deren Anwendung ausprobieren und Fragen individuell beantwortet bekommen.

Zu der Tagung waren auch die OP-Personal-Teams eingeladen, die teilweise mit den Ärzten diskutierten, teilweise in parallelen Sitzungen ihre Kurse planten und Inhalte verbesserten sowie Erfahrungen zwischen den Ländern austauschten.

Insgesamt fand der Austausch zwischen den ältesten und aktivsten Sektionen der AO in herzlicher Atmosphäre und mit sehr konstruktivem, kollegialem Dialog statt.

Vor der Tagung trafen sich zum ersten Mal auch die Präsidien der 3 Länder, diskutierten gemeinsame Projekte und beschlossen, die Zusammenarbeit auf diversen Gebieten zu vertiefen. So findet ein gemeinsames MasterSymposium am 18. und 19. November 2019 in Wien statt mit dem Thema „Komplikationen und Infektionen: Was ist schief gelaufen?" Der deutschsprachige D-A-CH-Masterkurs 2018 in Davos war leider nur zu rund 65\% gebucht und pausiert in 2019. Zur Diskussion mit AOTrauma International steht, diesen Kurs in Zukunft nur jedes 2. Jahr in Davos stattfinden zu lassen.

Auf der Tagung wurde auch das AOTrauma-D-A-CH-Reisestipendium 2019 an Frau Dr. Monique Kribus, Jena verliehen, die dieses für einen Aufenthalt bei Heinz Bürger, Klagenfurt nutzen wird.

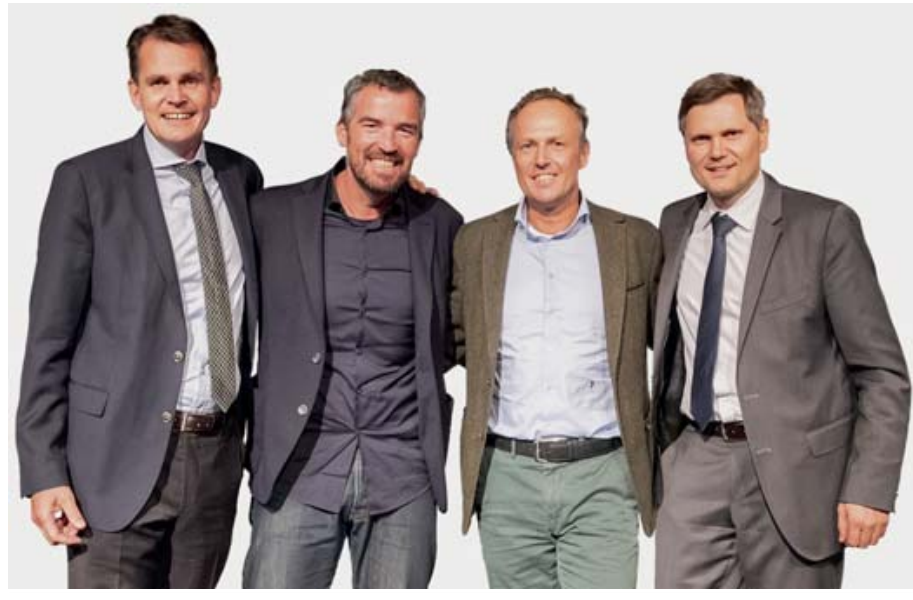

- Abb. 3 Die Präsidenten Stöckle (D), Borens (CH) und Kralinger (A) sowie Gastgeber Frosch. Quelle: AOTrauma

\section{Bilanz 2018 auf der \\ Mitgliederversammlung}

Auf der Mitgliederversammlung der deutschen AOTrauma beurteilte der neue Präsident Ulrich Stöckle 2018 insgesamt als ein schwieriges, aber erfolgreiches Jahr. Die Staffelstabübergabe vom alten zum neuen Präsidium war perfekt und extrem harmonisch. In den Gremien herrscht eine tolle Stimmung. Die Mitgliederzahl wächst stetig und liegt aktuell bei 835 .

Von den 15 AOTD-Kursen für Ärzte waren 11 ausgebucht mit insgesamt 944 Teilnehmern (96\% Auslastung). Premiere hatte der Schulter-Kurs in Ulm. Die 10 AOTD-Seminare wurden von 528 Teilnehmern besucht. Erstmalig 


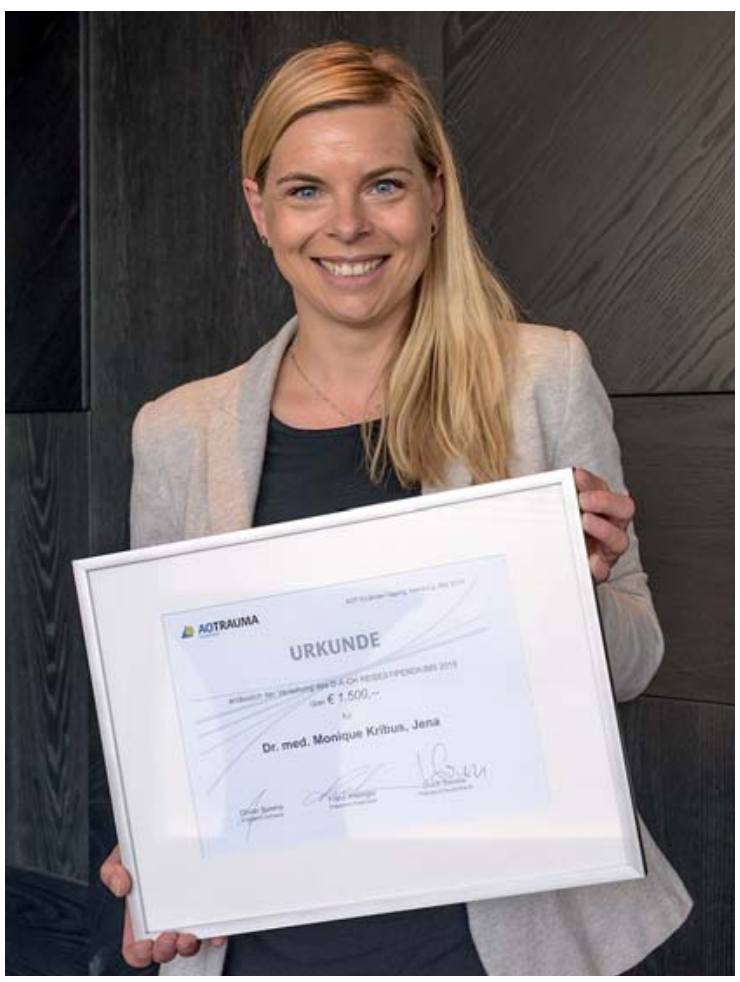

Abb. 4 Dr. Kribus mit der Urkunde. Quelle: AOTrauma

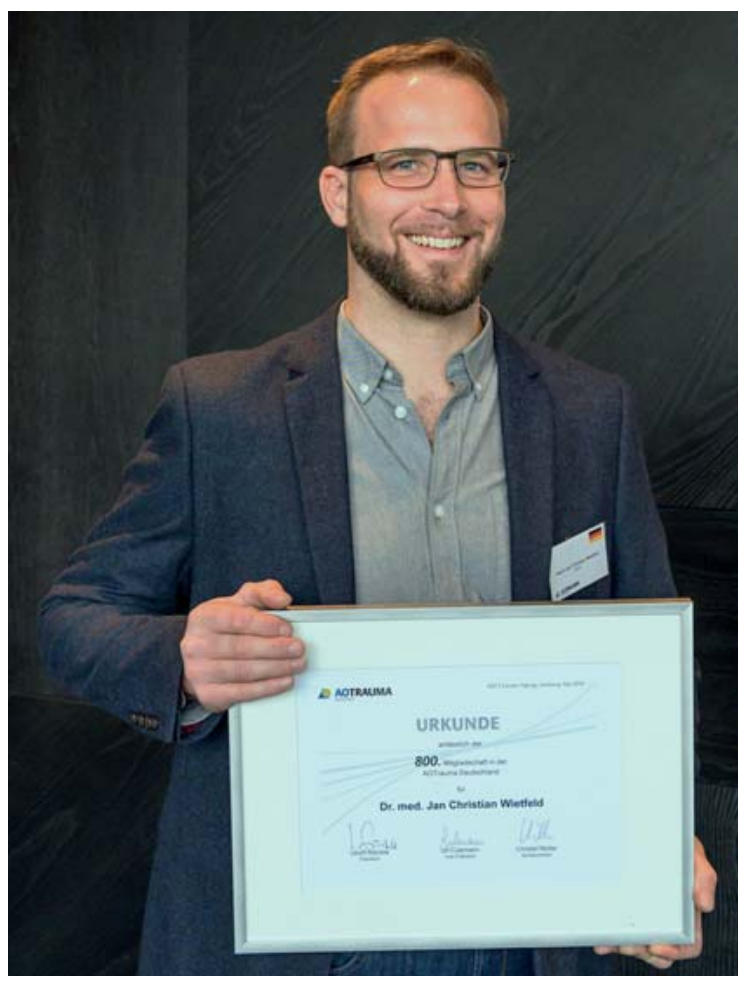

Abb. 5 800. AOTD-Mitglied Jan Wietfeld. Quelle: AOTrauma

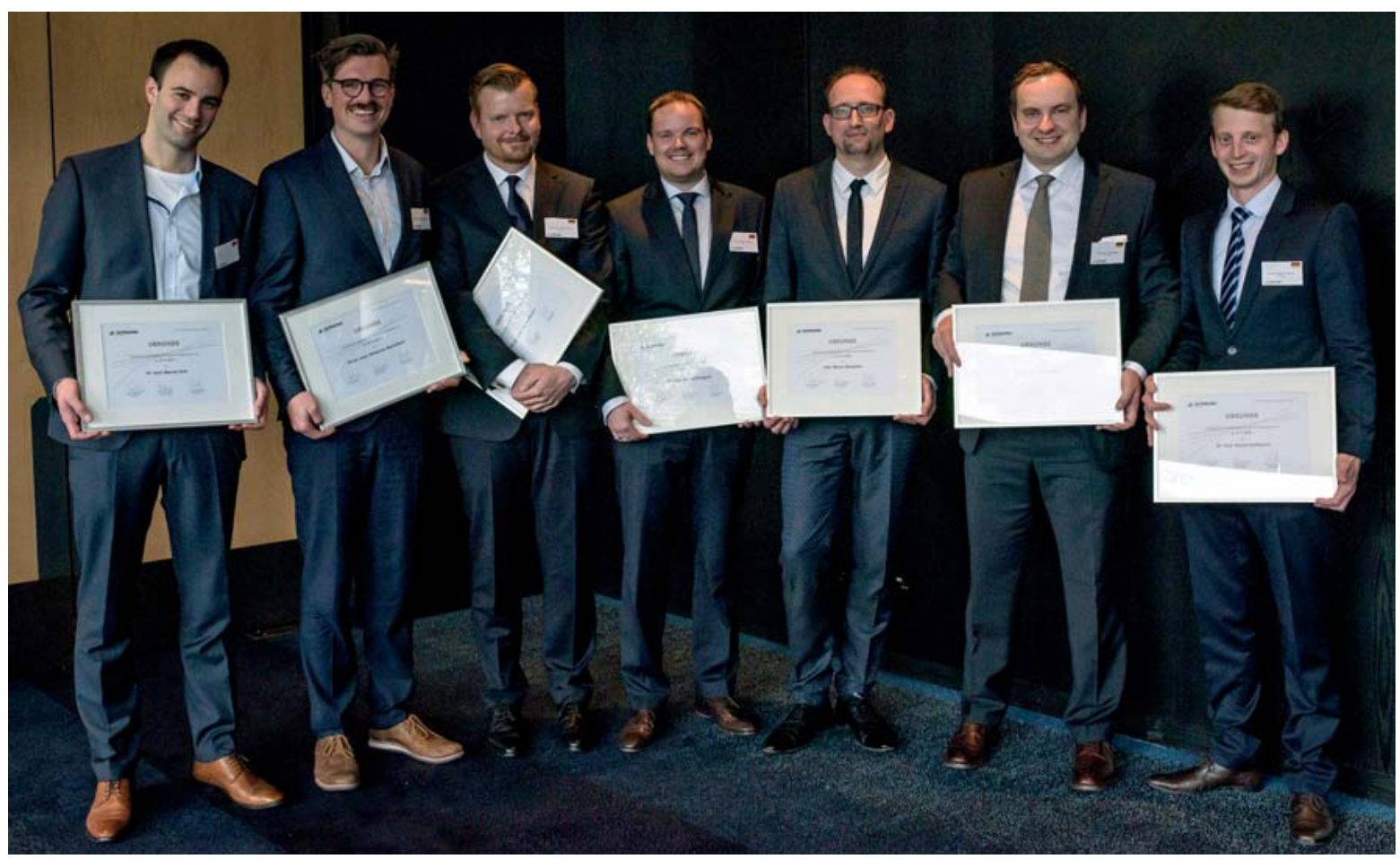

Abb. 6 Die 7 Reisestipendiaten. Quelle: AOTrauma 


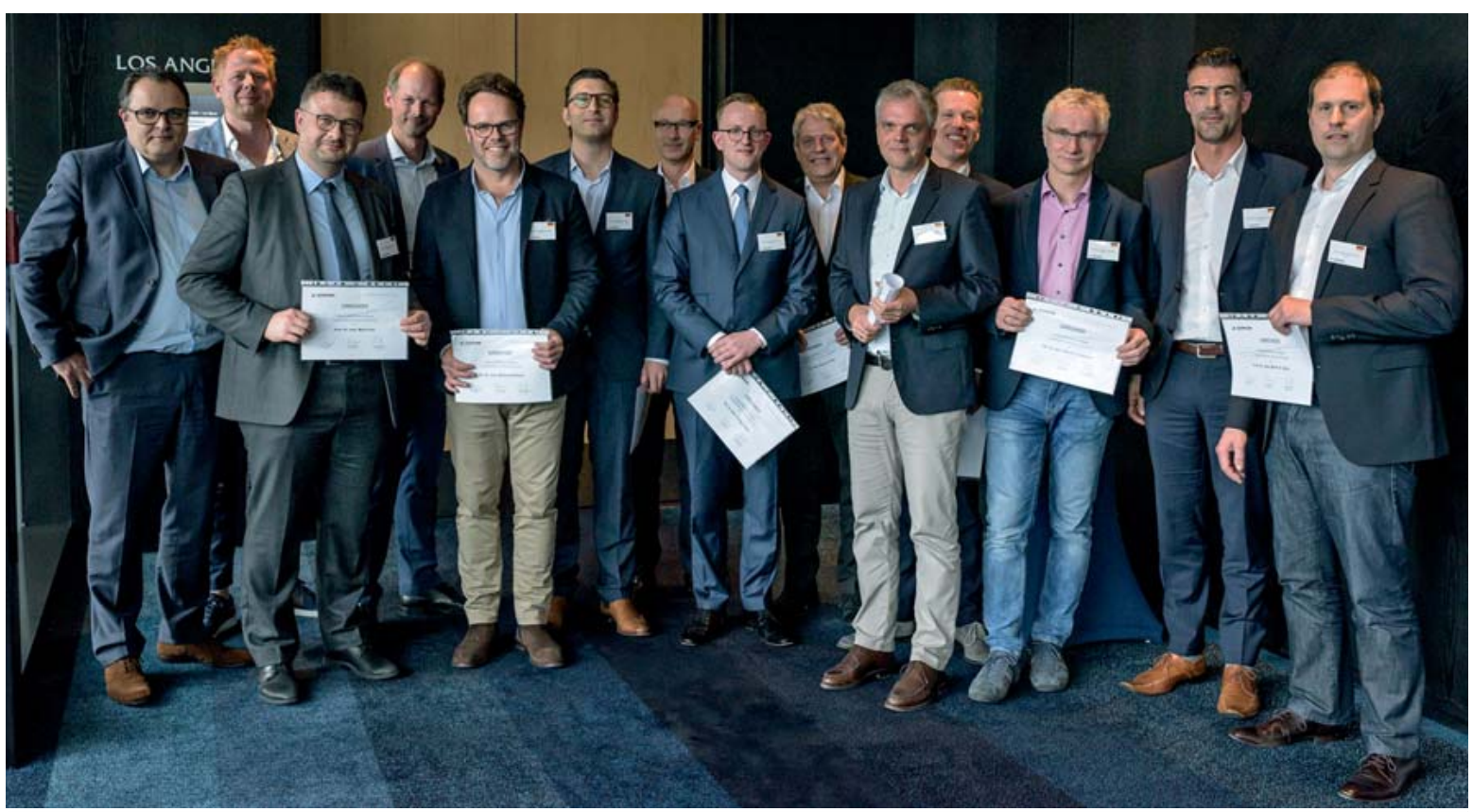

- Abb. 7 Die neuen Akademiemitglieder. Quelle: AOTrauma

fanden Seminare in Oldenburg und Fulda statt. Die Teilnehmerzahlen der ORP-Veranstaltungen sind 2018 deutlich gestiegen: 7 Kurse mit 424 Teilnehmern (in 2017: 370) und drei OP-Tage mit 167 Teilnehmern (in 2017: 118).

Problematisch war die Umsetzung der neuen Datenschutz-Richtlinie. Die postalische Bewerbung der Veranstaltungen musste zeitweilig eingestellt werden. Die Auswirkungen in Form von gesunkenen Teilnehmerzahlen waren gegen Ende 2018 und Anfang 2019 deutlich erkennbar.

Knapp 200 deutsche Ärzte haben mittlerweile das AO Faculty Education Program (FEP) absolviert. Aber beim Chairman Education Program (CEP) hat die AOTD Nachholbedarf, denn von ca. 100 Kurs- und Seminarleitern haben erst gut 20 das CEP absolviert. Deshalb fand 2018 zum ersten Mal ein Chairman Education Program (CEP) in Deutschland statt, was im November 2019 wiederholt wird.

Die Regel: „AO Faculty muss AOTrauma-Mitglied sein“ ist mittlerweile vollständig akzeptiert.

Von den 144 AOT Fellowship-Zentren weltweit befinden sich 31 in Deutschland. 61 der 290 Fellows weltweit in 2018 wählten sich Deutschland als Zielort. Neun Deutsche gingen ins Ausland. Es könnten sich mehr deutsche AOT-Mitglieder auf eine Fellowship bewerben, insbesondere die „Visit-the-Expert“ Fellowship für erfahrene Chirurgen wird bisher noch viel zu selten genutzt.

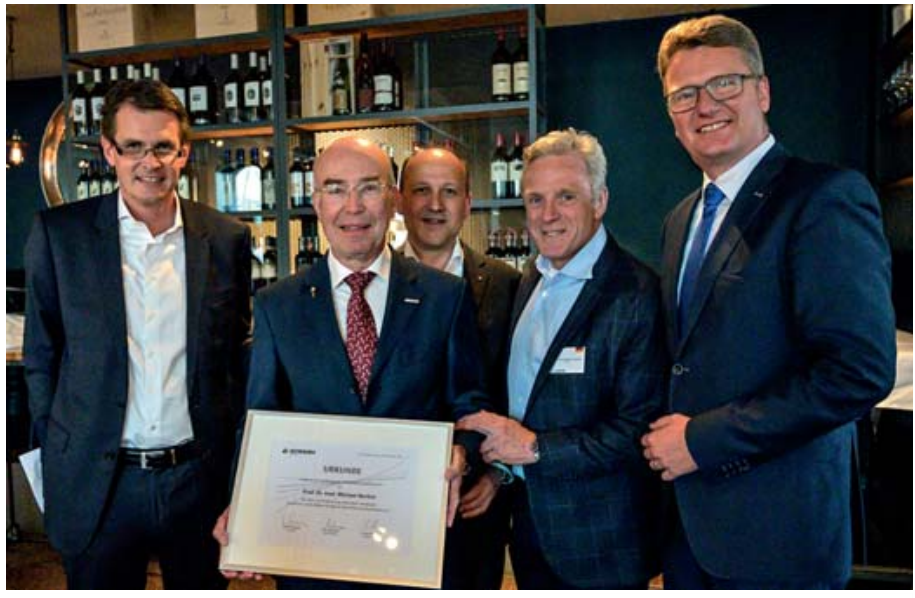

- Abb. 8 Michael Nerlich und das Präsidium. Quelle: AOTrauma

Für das deutsche AOT-Reisestipendium gab es 23 Bewerbungen, was einen Rekord darstellt. Die Auswahlkommission, bestehend aus Frosch, Fuchs, Hofmann und Stuby entschied sich für Baumbach (München), Bäumlein (Marburg), Burggraf (Essen), Graßmann (Düsseldorf), Hoffmann (Göttingen), Orth (Homburg/Saar) und Stübig (Hannover). 


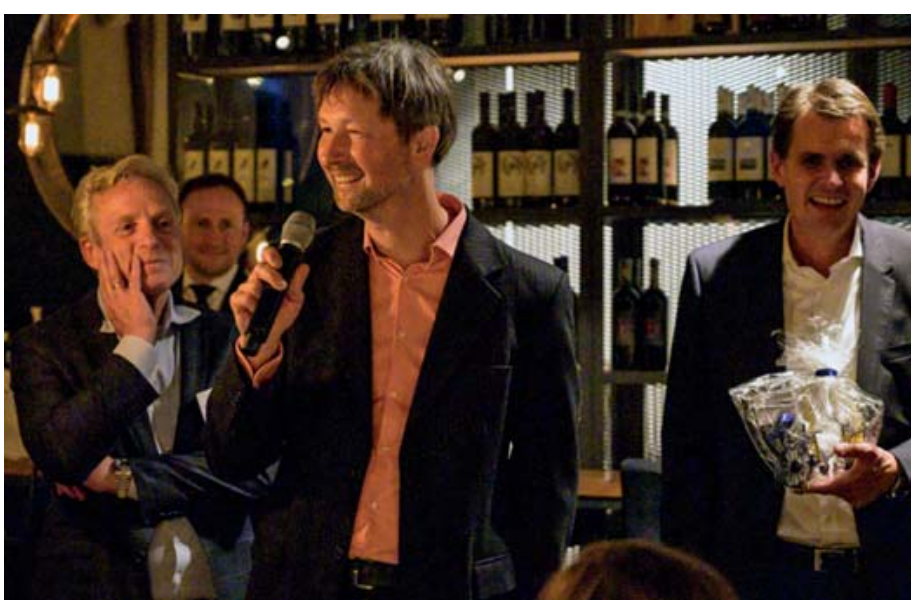

- Abb. 9 Wilbrandt verabschiedet sich von den Mitgliedern. Quelle: AOTrauma

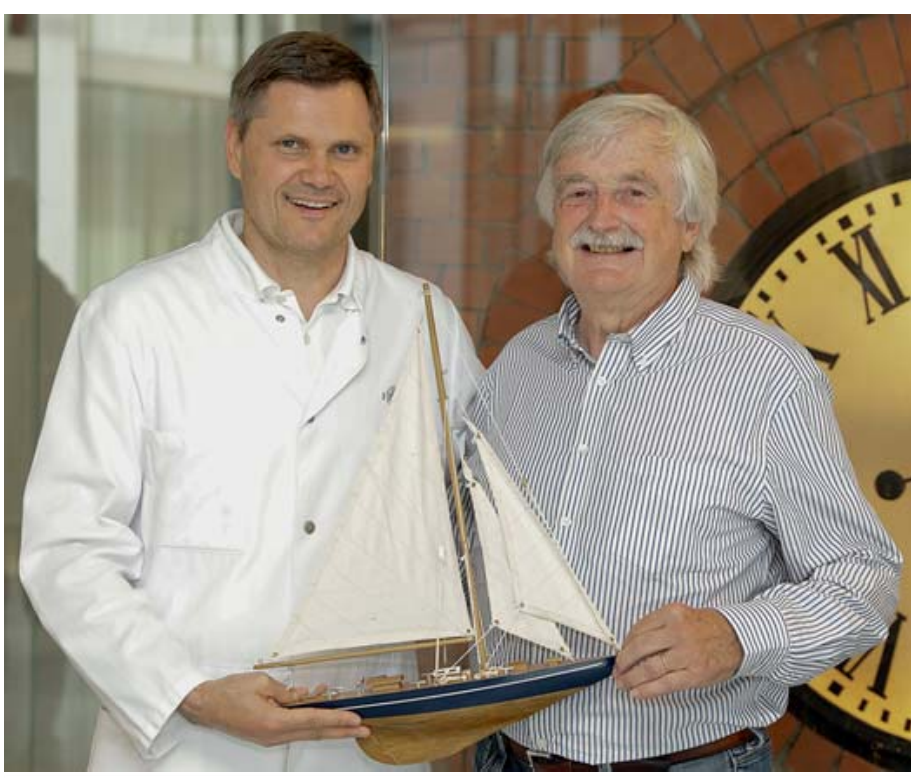

- Abb. 10 Gastgeber Karl-Heinz Frosch überreicht Spende an Heiner Winker. Quelle: AOTrauma

\section{Neu aufgenommene Akademiemitglieder}

Das Präsidium verlieh den 14 neu aufgenommenen Akademiemitgliedern die Aufnahmeurkunde. Die neuen Mitglieder der Akademie sind Hammer (Freiburg), Hartel (Hamburg), Krüger (Siegen), Lichte (Aachen), Meller (Hannover), C. Müller (HH-Wandsbek), M. Müller (Ibbenbühren), Ochs (Konstanz), Perl (Erlangen), Priemel (Ham-
burg-Eppendorf), Pütz (Bonn), Roetman (Rheine), Sander (Borken) und van Schoonhoven (Bad Neustadt).

\section{Ehrenmitgliedschaft}

Auf dem stimmungsvollen Festabend wurde Michael Nerlich, Regensburg zum AOTD-Ehrenmitglied ernannt.

\section{Wechsel in der AOTD-Geschäftsstelle}

Zur Mitte des Jahres wird es einen Wechsel in der Leitung der Geschäftsstelle geben. Philip Wilbrandt verlässt nach $41 \frac{1}{2}$ Jahren die AOTD auf eigenen Wunsch. Sein Nachfolger wird George Clay (siehe separaten Artikel in diesem Heft).

\section{Segeln für Nepal}

Nach Abschluss der Tagung luden Wolfgang Lehmann und Philip Wilbrandt zum „AO Family \& Friends“ Event im Barca Club an der Außenaster ein. Es kamen weit über 70 Personen zum „Grillen und Chillen“. Höhepunkt war eine Segelregatta, an der 38 Personen teilnahmen, darunter 7 Ehefrauen und 6 Kinder. Souveräner Sieger wurde das Team vom UKE mit Max Hartel als Steuermann. Das Team „Traumaschiff“ mit Richard Sellei wurde mit dem „Trau-ma-növer“-Pokal für die spektakulärste Wende ausgezeichnet. Dank des Startgeldes und weiterer Spenden kamen 1150,- EUR zusammen, die dem Dhulikhel Hospital - Kathmandu University Hospital in Nepal gespendet wurden. Damit wird - zusammen mit weiteren von Heiner Winker eingeworbenen Mitteln ein Hygieneprojekt finanziert: die Ausstattung einer neu zu bauenden Krankenhauswäscherei mit Waschmaschinen und Trocknern, Einweisung des Personals und Unterhalt der Technik im Sinne der Nachhaltigkeit.

Ein herzlicher Dank geht an das Team vom Barca Club an der Alster.

Die nächste AOTrauma D-A-CH 3-Länder Tagung findet 2022 in Wien statt. Im kommenden Jahr lädt die AOTrauma Deutschland zur Jahrestagung am 8. und 9. Mai 2020 nach Mainz.

Prof. Dr. Ulrich Stöckle, Berlin

Prof. Karl-Heinz Frosch, Hamburg

Bibliografie

DOI https://doi.org/10.1055/a-0953-5478

OP-JOURNAL 2019; 35: 192-196 @ Georg Thieme Verlag KG Stuttgart · New York ISSN 0178-1715 